\title{
Compressive Sensing Based Channel Feedback Protocols for Spatially-Correlated Massive Antenna Arrays
}

\author{
Ping-Heng Kuo* $^{*}$ H. T. Kung ${ }^{\dagger}$ Pang-An Ting* \\ * Information and Communications Research Laboratories, Industrial Technology Research Institute (ITRI) \\ Hsinchu, Taiwan. Email: \{pinghengkuo, pating\}@itri.org.tw \\ $\dagger$ School of Engineering and Applied Sciences, Harvard University \\ Cambridge, MA, USA. Email: kung@harvard.edu
}

\begin{abstract}
Incorporating wireless transceivers with numerous antennas (such as Massive-MIMO) is a prospective way to increase the link capacity or enhance the energy efficiency of future communication systems. However, the benefits of such approach can be realized only when proper channel information is available at the transmitter. Since the amount of the channel information required by the transmitter is large with so many antennas, the feedback is arduous in practice, especially for frequency division duplexing (FDD) systems. This paper proposes channel feedback reduction techniques based on the theory of compressive sensing, which permits the transmitter to obtain channel information with acceptable accuracy under substantially reduced feedback load. Furthermore, by leveraging properties of compressive sensing, we present two adaptive feedback protocols, in which the feedback content can be dynamically configured based on channel conditions to improve the efficiency.
\end{abstract}

\section{INTRODUCTION}

A new paradigm of multi-antenna techniques dubbed as Massive-MIMO, which equips the wireless transceiver with numerous antennas, has been put forward as a technology option for realizing high-throughput green radios in beyond $4 \mathrm{G}$ wireless communication systems [1]. A tremendous array gain can be attained by steering the power emitted from a large number of transmit antennas to the targeted direction (beamforming), which in turn increases the link capacity significantly under a fixed transmission power, or equivalently, dramatically decreases the required transmission power to maintain the desired quality of service (QoS). Moreover, Massive-MIMO is a promising approach for multi-user communications in future cellular networks [2].

However, to harvest the potential benefits of MassiveMIMO, the transmitter must acquire the instantaneous channel state information (CSI). By exploiting channel reciprocity, CSI acquisition at the transmitter is more feasible under time division duplexing (TDD) operation [2], [3], [4]. With frequency division duplexing (FDD), channel estimation becomes much more challenging, and a dedicated feedback mechanism for the receiver to report CSI is needed. Even if the CSI can be perfectly estimated at the receiver, the required bandwidth for CSI feedback is aggravated as the number of transmit and receive antennas increases. Nonetheless, FDD is generally considered to be more effective for systems with symmetric traffic or delay-sensitive applications.

This paper studies the feasibility of alleviating the heavy CSI feedback load in Massive-MIMO schemes. Conventional CSI feedback reduction methods, such as vector quantization or codebook-based approaches [5], may not be suitable for Massive-MIMO as the codebook size has to be expanded extensively (which leads to heavier feedback overhead) to capture fine-grain spatial channel structures. Otherwise, the resultant quantization errors may not be acceptable for schemes that are sensitive to inaccuracy in CSI estimates. This makes codebook design for Massive-MIMO much more difficult.

Recently, the theory of compressive sensing (CS) [6], [7] has been applied in diverse contexts of signal processing and communications, where the information content is sparse. Via random projections, CS can efficiently encode this sparsity without having to know the location of the information. In [8], CS has been employed for the receiver to report channel qualities of OFDM subcarriers. Also, both [9] and [10] have leveraged CS to support feedback protocols for opportunistic multi-user MIMO downlink transmission. Unlike the aforementioned prior work, this paper uses a different insight that strong spatial correlations are expected in massive closelypacked antenna arrays, so the channel can be represented with a sparse form in the spatial-frequency domain. Thus, the theory of CS can be applied to develop an efficient compression mechanism for CSI feedback, which still allows CSI recoveries with acceptable accuracy at the transmitter. Based on such feedback mechanism, we introduce two novel adaptive feedback protocols improving further the feedback efficiency for systems with massive antenna arrays.

The main contributions of this paper are summarized as follows:

1) We use CS to reduce the CSI feedback load in a Massive-MIMO system by exploiting the expected sparsity in the spatial-frequency domain resulting from spatially-correlated antenna arrays. To our knowledge, we are the first in the literature to suggest such a feedback load reduction approach.

2) We corroborate by simulations that, with the CS-based method, small compression ratios can be achieved in CSI feedback reduction for spatially-correlated Massive- 
MIMO schemes (Figure 2).

3) We propose an adaptive CS-based feedback scheme, where the feedback can be dynamically configured based on channel conditions to improve the efficiency.

4) We propose a hybrid adaptive feedback scheme which can leverage the optimality property of the KarhunenLoeve Transform (KLT) in spite of its signal-dependent nature.

5) We show by simulation that CS-based feedback can achieve near optimal rank-1 beamforming performance (Figure 7), and the hybrid adaptive feedback can achieve the same for slow-varying channels, while incurring substantially reduced feedback load (Section IV-B).

The remainder of this paper is organized as follows. Section II provides the system model, as well as a review of CS operation. In Section III, CS is applied to the design of the channel feedback mechanism. Two adaptive feedback protocols are proposed in Section IV. Finally, a conclusion is drawn in Section V.

\section{BACKGROUND AND ASSUMPTIONS}

\section{A. System Model}

This paper considers a point-to-point Massive-MIMO wireless communication system with $N_{t}>>1$ transmit antennas and $N_{r} \geq 1$ receive antennas. The $N_{r} \times N_{t}$ spatially-correlated MIMO channel matrix can be modeled as:

$$
\boldsymbol{H}=\frac{1}{\sqrt{\operatorname{tr}\left(\boldsymbol{R}_{R X}\right)}} \boldsymbol{R}_{R X}^{\frac{1}{2}} \boldsymbol{H}_{i i d} \boldsymbol{R}_{T X}^{\frac{1}{2}},
$$

where $\boldsymbol{H}_{\text {iid }}$ is an $N_{r} \times N_{t}$ matrix with independent and identically distributed (i.i.d.) complex zero-mean, unit variance, Gaussian random entries; $\boldsymbol{R}_{T X}$ and $\boldsymbol{R}_{R X}$ are the correlation matrices at the transmitter side and receiver side, respectively. We assume that uniformly-spaced linear antenna arrays are installed at both ends of the radio link, and the spatial correlations are solely governed by the Jakes model often used in the literature, so any entry of $\boldsymbol{R}_{T X}$ or $\boldsymbol{R}_{R X}$ (the correlation coefficient between the $i^{t h}$ and the $j^{t h}$ antenna within a transmit or receive array), is

$$
r_{i j}=J_{0}\left(\frac{2 \pi d_{i j}}{\lambda}\right)
$$

where $d_{i j}$ is the distance between the two antennas, $\lambda$ is the carrier wavelength, and $J_{0}(\cdot)$ denotes the zeroth-order Bessel function of first kind. Given that the scope of this paper is focussed on CSI compression, we assume $\boldsymbol{H}$ can be perfectly estimated at the receiver, and certain channel information, such as $\boldsymbol{H}$ itself or any spatial signature extracted from $\boldsymbol{H}$, can be sent to the transmitter via an ideal (error-free) feedback link. For instance, the beamforming vectors can be computed from $\boldsymbol{H}$ by singular value decomposition (SVD):

$$
\boldsymbol{H}=\boldsymbol{U} \boldsymbol{\Sigma} \boldsymbol{V}^{\dagger},
$$

where both $\boldsymbol{U}$ and $\boldsymbol{V}$ are unitary matrices, while $\boldsymbol{\Sigma}$ is a diagonal matrix. Due to the rank-reducing nature of the heavily correlated MIMO channel, it is appropriate to send the data signal only on the strongest spatial link (rank-1 beamforming) in order to maximize link capacity. In such cases, the first column of $\boldsymbol{V}$ (denoted as $\boldsymbol{V}_{1}$ ) is the only channel information required by the transmitter. Nevertheless, in certain applications (such as zero-forcing beamforming [11] and interference alignments [12]), the knowledge on full MIMO channel matrix $\boldsymbol{H}$ is needed at the transmitter. Note that the correlations among entries of channel matrix $\boldsymbol{H}$ also result in correlations among the elements of $\boldsymbol{V}_{1}$, which implies that both $\boldsymbol{H}$ and $\boldsymbol{V}_{1}$ can be sparsified in the spatial-frequency domain under certain transformations. Thus, we note that the CS-based feedback methods we propose in this paper are applicable to both $\boldsymbol{H}$ and the principal beamforming vector $\boldsymbol{V}_{1}$. Without loss of generality in the algorithmic procedures, we focus on $\boldsymbol{H}$ as the target signal of the proposed feedback methods.

\section{B. Review of Compressive Sensing}

Compressive sensing (CS) encoding is capable of capturing the $K$ non-zero components of a $K$-sparse $N \times 1$ signal vector, where $K<<N$, by compressing the signal into $M<<N$ measurements via random projections. Nonetheless, in many practical cases, the $N \times 1$ target signal (denoted as $x$ ) does not exhibit any sparsity by itself until it undergoes a sparsifyingtransformation:

$$
S=\Psi x
$$

where $S$ is the sparse representation of $x$, and $\Psi$ is an $N \times N$ sparsifying-basis. The quintessential examples of $\boldsymbol{\Psi}$ include discrete cosine transform (DCT) matrix, discrete Fourier transform (DFT) matrix, etc. Such transformations are usually orthogonal:

$$
x=\Psi^{T} S,
$$

where $(\cdot)^{T}$ represents matrix transpose. In contrast, conventional data compression schemes, such as entropy-based compression, simply encode the data signal as $S$. In these cases, the information on indices (positions) of the non-zero elements in $S$ should be computed and also be jointly encoded for purposes of data restoration at the decoder. With CS, there is no such overheads; the target signal $x$ is blindly encoded as an $M \times 1 \mathrm{CS}$ measurement vector, $y$ [7]:

$$
y=\boldsymbol{\Phi} \boldsymbol{\Psi}^{T} S=\boldsymbol{\Phi} x,
$$

where $\boldsymbol{\Phi}$ is an $M \times N$ measurement matrix, the elements of which are random variables generated in accordance to distributions such as Gaussian or Bernoulli. To reconstruct the signal $x$ from $y$, the decoder needs to solve an underdetermined linear system since there are more unknowns $(N)$ than equations $(M)$. This can be formulated as the following $l_{1}$ norm minimization problem:

$$
\min \|S\|_{l_{1}} \quad \text { s.t. } \quad y=\boldsymbol{\Phi} \Psi^{T} S,
$$

which typically could be solved by optimization algorithms such as linear programming (LP), basic pursuit (BP), and orthogonal matching pursuit (OMP). Note that $S$ can be recovered with a high probability as long as $M$ is sufficiently 
large ${ }^{1}$ and $\boldsymbol{\Phi} \Psi^{T}$ satisfies restricted isometry property (RIP) [6]. Finally, the target signal, $x$, can be reconstructed as $\hat{x}$ via (5) once $S$ becomes available from solving (7).

\section{MASSIVE-MIMO CHANNEL FEEDBACK BASED ON COMPRESSIVE SENSING}

We assume that in a Massive-MIMO system, antenna arrays at both transmitter and receiver are on the same platforms and closely-packed. Due to correlations among the antennas on each platform, it is expected from signal processing theory that the channel information has a sparse representation in the spatial-frequency domain. Based on this insight, instead of sending $\boldsymbol{H}$ on feedback directly, the CS techniques delineated in Section II-B could be applied for feedback compression. Note that the common operations are carried out separately for real and imaginary parts of the MIMO channel matrix $\boldsymbol{H}$ in all proposed methods of this paper. For the sake of convenience, the notation $\widetilde{\boldsymbol{H}}$ is used to denote the target signal in rest of the paper, which represents either the real part or imaginary part of $\boldsymbol{H}$.

As aforementioned, we assume that the receiver has perfect knowledge on $\boldsymbol{H}$, which should be ideally shared with the transmitter through feedback, for purposes of appropriate transmitter configurations. In order to reduce the radio resources required by the feedback, the information of $\widetilde{\boldsymbol{H}}$ is compressed into $M$ measurements via the CS operation. As the initial setting, $\widetilde{\boldsymbol{H}}$ should first be vectorized into an $N_{r} N_{t} \times 1$ vector $^{2}$ :

$$
h=\operatorname{vec}(\widetilde{\boldsymbol{H}}) .
$$

Following (6), $h$ is encoded into a measurement vector (by random projections) as the compressed feedback content:

$$
y=\boldsymbol{\Phi} h .
$$

Thus, an $N_{r} N_{t} \times 1$ channel vector $(h)$ is compressed into an $M \times 1$ measurement vector ( $y$ ) with (9). As we will see due to the expected sparsity in the spatial-frequency domain, we can make $M$ to be much smaller than $N_{r} N_{t}$, while allowing CSI reconstruction at the transmitter to satisfy the required accuracy. The feedback load is thereby reduced by a compression ratio of $\eta=M /\left(N_{r} N_{t}\right)$. The elements of $\boldsymbol{\Phi}$ are randomly generated offline based on Gaussian distributions, so both transmitter and receiver are aware of $\boldsymbol{\Phi}$ with preconfigurations. Thus, the transmitter is able to recover the channel information, $\hat{h}$, based on (7) and (5) once it acquires $y$ from the feedback. In simulations, this paper adopts OMP [13] as the optimization algorithm to solve (7) due to its relatively low computational complexity. The schematic of the proposed CS-based feedback mechanism is illustrated in Figure 1. The choice of the sparsifying-basis, $\boldsymbol{\Psi}$, plays a key role in performance of the recovery, i.e., the error magnitude of the recovered CSI under a given compression

\footnotetext{
${ }^{1}$ Theoretically, the size of $y$ should satisfy $M>c K \log (N / K)$, where $c$ is a small constant. A rule of thumb is to set $M$ as two to four times of $K$.

${ }^{2}$ This step of vectorization can be omitted if the target signal is a vector originally, e.g., $\boldsymbol{V}_{1}$ or a multiple-input single-output (MISO) channel vector.
}

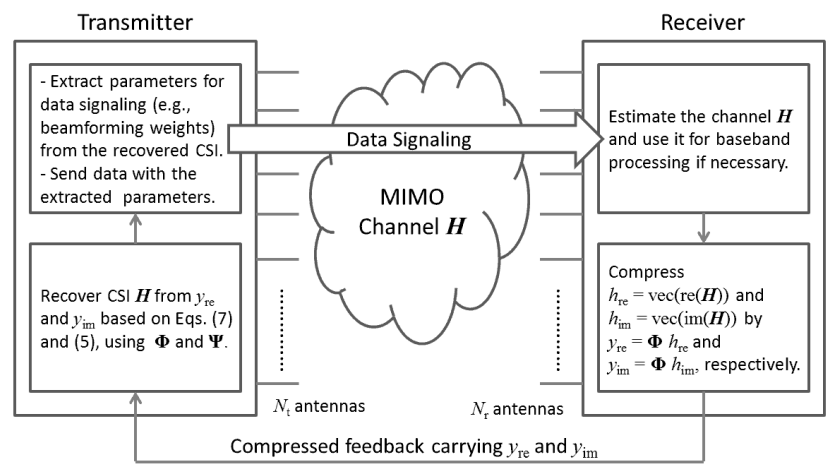

Fig. 1. The schematic of the proposed CS-based MIMO channel feedback method, where $\operatorname{re}(\boldsymbol{H})$ and $\operatorname{im}(\boldsymbol{H})$ represent the real and imaginary parts of $\boldsymbol{H}$, respectively.

ratio. Generally speaking, it is desirable to select a sparsifyingbasis that provides a more sparse representation (fewer nonzero elements in $S$, or smaller $K$ ) of $h$. In this paper, two such bases are considered: two-dimensional discrete cosine transform (2D-DCT) and Karhunen-Loeve transform (KLT).

\section{A. Two-Dimensional Discrete Cosine Transform}

As mentioned earlier, the elements of $\widetilde{\boldsymbol{H}}$ are expected to be strongly correlated in both dimensions. In order to realize a sparse representation of $\widetilde{\boldsymbol{H}}$ in the spatial-frequency domain by fully exploiting such correlation structure, 2DDCT can be employed as the sparsifying-basis $\Psi$. Note that the matrix operation of applying 2D-DCT on $\widetilde{\boldsymbol{H}}$ can be written as $\boldsymbol{C}_{N_{r}}^{T} \widetilde{\boldsymbol{H}} \boldsymbol{C}_{N_{t}}$, where $\boldsymbol{C}_{L}$ is the $L \times L$ DCT matrix. Because DCT matrix is a 2D-separable sparsifying-basis [14], the sparse representation of $h$ is

$$
S=\left(\boldsymbol{C}_{N_{t}} \otimes \boldsymbol{C}_{N_{r}}\right)^{T} \operatorname{vec}(\widetilde{\boldsymbol{H}})=\left(\boldsymbol{C}_{N_{t}} \otimes \boldsymbol{C}_{N_{r}}\right)^{T} h,
$$

where $\otimes$ is Kronecker product. As $h=\left(\boldsymbol{C}_{N_{t}} \otimes \boldsymbol{C}_{N_{r}}\right) S$, from (4) it is easy to see that the sparsifying-basis for $\mathrm{CS}$ recovery with 2 D-DCT is $\boldsymbol{\Psi}^{T}=\left(\boldsymbol{C}_{N_{t}} \otimes \boldsymbol{C}_{N_{r}}\right)$.

\section{B. Karhunen-Loeve Transform (KLT)}

It can be shown that KLT provides the optimal sparse representation with only one non-zero element $(K=1)$ [15], [16], which promises accurate CS recoveries even if only a small number of measurements are available. Given that we have the instantaneous $N_{r} N_{t} \times N_{r} N_{t}$ correlation matrix of $h$, written as:

$$
\boldsymbol{W}=h h^{T},
$$

the KLT sparsifying-basis, $\boldsymbol{Q}$, can be computed by eigenvalue decomposition:

$$
\boldsymbol{W}=\boldsymbol{Q} \Lambda \boldsymbol{Q}^{T}
$$

where $Q$ is comprised of eigenvectors of $\boldsymbol{W}$, while the diagonal entries of $\boldsymbol{\Lambda}$ are the correspondent eigenvalues.

For a Massive-MIMO system with $N_{t}=N_{r}=32$, a uniform antenna-spacing $(d)$ of $0.01 \mathrm{~m}$, and a carrier frequency of $700 \mathrm{MHz}$, we compared the recovery performance with $\boldsymbol{\Psi}$ based on 2D-DCT and KLT, under different compression 


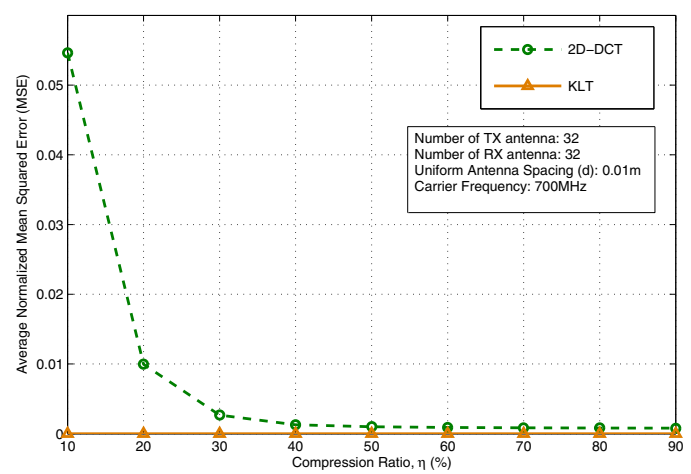

Fig. 2. The average normalized MSE of CS-based MIMO channel feedback recovery using 2D-DCT and KLT basis under different compression ratios. For the KLT results, we assume that $\boldsymbol{Q}$ is perfectly known at the transmitter.

ratios. The accuracy metric is the average normalized mean squared error (MSE) between the ground-truth $h$ and the computed $\hat{h}$. For ideal cases where $\boldsymbol{Q}$ is perfectly known at the transmitter, Figure 2 shows that exact recovery can be achieved with KLT under any compression ratio. However, 2DDCT can be a worthy approach for less stationary signal, given that its $\boldsymbol{\Psi}$ is signal-independent. We note that under 2D-DCT the resultant MSE is only $1 \%$ even with a low compression ratio of $20 \%$. On the other hand, given that its basis is signaldependent, the KLT approach can be attractive for relatively stationary target signals; its sparsifying-basis could be tracked more easily in these scenarios.

\section{FeEdBACK with AdAptive FeEdBack Protocols}

Based on the Massive-MIMO channel feedback mechanism proposed in Section III, we developed two different adaptive feedback protocols, in which the feedback can be dynamically configured based on channel condition to improve the efficiency.

\section{A. Adaptive CS-based Feedback}

By inspecting Figure 2, a trade-off between recovery performance and feedback burden can be observed when 2DDCT is chosen as the sparsifying-basis. This motivated us to design a feedback scheme which uses a higher compression ratio only when the system performance becomes more sensitive to channel information inaccuracy at the transmitter. Otherwise, as long as the performance degradation caused by channel information recovery error is tolerable, a default lower compression ratio can be applied to reduce the radio resources required by feedback. Since the compression ratio, $\eta=M /\left(N_{r} N_{t}\right)$, is proportional to $M$, we adjust the value of $M$ at the receiver, in accordance to the instantaneous fading gain. When the channel gain is strong, a slight deviation in CSI due to a lower $\eta$ might not result in a severe performance loss; in this case the feedback can be reduced by using a lower default value of $M$. Conversely, when the channel is too weak to support robust transmission without highly accurate CSI, the receiver should boost the compression ratio by using a higher

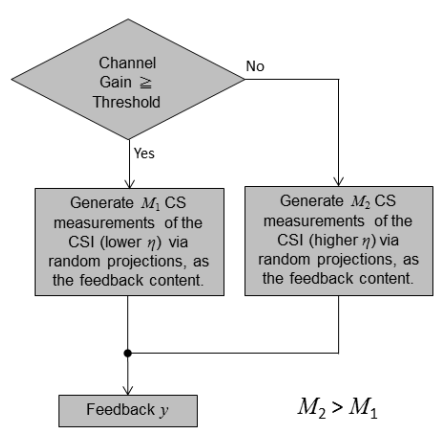

Fig. 3. The flow-chart of the CS-based feedback scheme with adaptive compression ratio.

default value of $M$ when preparing the feedback. Therefore, the transmitter can configure the physical layer parameters in a more accurate manner, at the expense of heavier feedback overhead. In its simplest form, this is essentially a binary adaptation, in which the compression ratio is switched between two possible levels. Note that the extension to adaptation among more than two levels is also possible, where the channel gain range is partitioned into several regions, and each region corresponds to a specific compression ratio.

Consider a scenario where $M$ is altered between $M_{1}$ and $M_{2}\left(M_{1}<M_{2}\right)$, and $\boldsymbol{\Phi}$ is an $M_{2} \times N_{t} N_{r}$ random measurement matrix stored at both transmitter and receiver. When the lower compression ratio is used $\left(M=M_{1}\right)$, only the first $M_{1}$ rows of $\boldsymbol{\Phi}$ are leveraged for compression and reconstruction at the receiver and transmitter, respectively. If $M=M_{2}$, then the full matrix of $\boldsymbol{\Phi}$ is applied for computation. Thus, apart from the CS measurements, the feedback content should also include an indication of $M$ (an 1-bit indication is enough for the binary adaptation case mentioned above), so the transmitter is able to determine an appropriate portion of $\boldsymbol{\Phi}$ for CSI recovery. The flow-chart of compression ratio selection for this binary adaptation scheme is depicted in Figure 3.

The simulation results in Figure 4 show the symbol error rates (SER) performance of rank-1 beamforming with Massive-MIMO systems under adaptive and non-adaptive CS-based feedback schemes, when 2D-DCT is used as the sparsifying-basis for channel recovery. It is apparent that the adaptive CS-based feedback scheme, which switches the compression ratio between $40 \%$ and $20 \%$ depending on whether the forward link gain exceeds some threshold level $\gamma$, will deliver better error-performance than the non-adaptive CSbased feedback scheme with a fixed compression ratio of $20 \%$. By setting $\gamma=18 \mathrm{~dB}$ in simulations, it results in a low probability of 0.143 in using the $40 \%$ compression ratio, while being able to achieve an improvement in SER. Note that a low signal-to-noise ratio (SNR) regime is considered in Figure 4 to reflect the energy efficiency benefits of MassiveMIMO schemes, the transmission power of which can be set relatively small. The proposed method could be extended to address lossy feedback, in which the additional measurements along with their row indices (assuming $\mathbf{\Phi}$ matrix has a large 


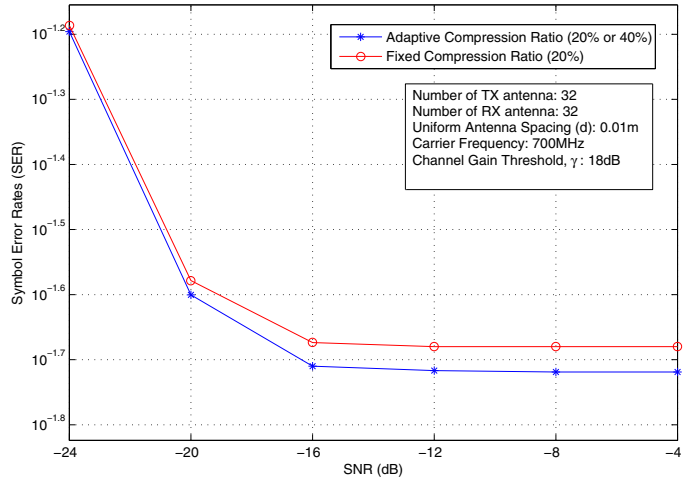

Fig. 4. Comparison on SER of rank-1 beamforming in Massive-MIMO with non-adaptive CS-based feedback (with a fixed compression ratio of $20 \%$ ) and adaptive CS-based feedback (with an adaptive compression ratio of $20 \%$ or $40 \%$ under a channel gain threshold $\gamma$ of $18 \mathrm{~dB}$ ).

number of rows) are continuously sent until the reception of an acknowledgement from the transmitter notifying that sufficiently many measurements have been received.

\section{B. Hybrid CS-KLT Adaptive Feedback}

From Section III-B, we know that KLT could be used to obtain the most compact representation of the underlying signal. The feedback load can be enormously relented if only the magnitude of the only non-zero element of $S$ (denoted as $s$ ) is sent. Due to the eigen-structure properties, it can be assumed that $s$ is always the first coefficient of $S$, so the feedback of its index is also not necessary. Nevertheless, with KLT, we still need the signal-dependent sparsifyingbasis $Q$ at the transmitter to recover the channel from $s$. In correspondence, we propose a CS-based feedback protocol that caters to slow-varying channels (such as indoor applications), which improves the feedback efficiency by allowing the system to switch the feedback content between CS measurements and $s$. The flow-chart of our proposal is illustrated in Figure 5.

Once the Massive-MIMO channel $\widetilde{\boldsymbol{H}}$ is estimated, the receiver should follow Section III to generate $y$ that consists sufficient CS measurements (based on a default value of $\eta$, which is associated to a large enough value of $M$ for CSI recovery with acceptably small errors (see Figure 2)). Prior to sending $y$ on feedback, the receiver emulates the recovery process that will be undertaken by the transmitter to compute the KLT basis, $\boldsymbol{Q}_{\text {est }}$, from the channel recovered by the emulation, $\hat{h}$. Upon acquiring $y$, the transmitter restores the CSI and extracts suitable parameter settings for data signaling. Additionally, the transmitter should also compute $\boldsymbol{Q}_{\text {est }}$ from the recovered channel $\hat{h}$. In the next signaling session, the receiver should first calculate the KLT-based sparse representation, $S$, of the newly estimated channel $\widetilde{\boldsymbol{H}}_{n e w}$. By presuming slow-varying channels, CSI could be approximated by using $\boldsymbol{Q}_{\text {est }}$ obtained in the preceding session:

$$
h_{t r y}=Q_{e s t}^{T} S \text {. }
$$

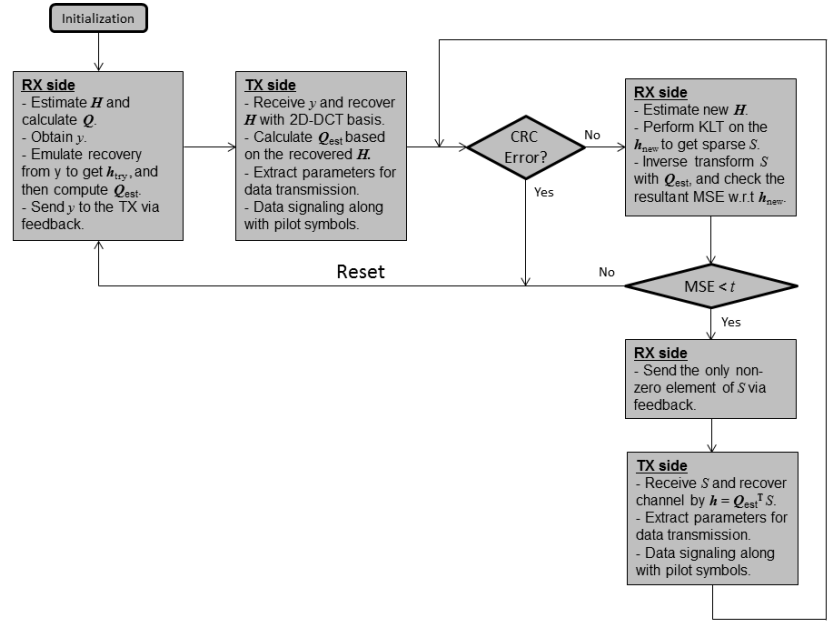

Fig. 5. The flow-chart of proposed hybrid CS-KLT adaptive feedback scheme.

Then, the timeliness of $\boldsymbol{Q}_{\text {est }}$ could be checked by examining the MSE of $\widetilde{\boldsymbol{H}}_{t r y}$ with respect to $\widetilde{\boldsymbol{H}}_{n e w}$, where $h_{t r y}=$ $\operatorname{vec}\left(\widetilde{\boldsymbol{H}}_{t r y}\right)$. If the MSE is low enough (below a threshold $t$ ), $\boldsymbol{Q}_{\text {est }}$ is not outdated and only $s$ would be sent by the feedback mechanism, so the transmitter could recover the channel by (13) as it also possesses $\boldsymbol{Q}_{\text {est }}$. Otherwise, if the MSE is too high and therefore unacceptable (higher than a threshold $t$ ), $\boldsymbol{Q}_{\text {est }}$ is deemed to be obsolete; and the feedback mechanism should be reset by updating the current $\boldsymbol{Q}_{\text {est }}$, which serves as an approximation to the KLT basis, at both the receiver and transmitter. In this case, a new CS measurement vector $y$ is prepared as the feedback content, analogous to the initial setup. Note that if a data transmission error is detected (for instance, the failure of cyclic redundancy check (CRC)) at the receiver, the feedback mechanism should also be reset. If the system has to reset in every signaling session, it is basically a non-adaptive CS-based feedback scheme.

This protocol reduces feedback by assuming that the channel is slow varying, so $\boldsymbol{Q}_{\text {est }}$ is reusable for $\alpha \geq 1$ consecutive subsequent sessions. Except for the first session upon reset, which requires the feedback of an $M \times 1$ measurement vector $y$, only a single entry $s$ is sent on feedback in each of the $\alpha$ remaining sessions. The accumulated feedback load over $1+\alpha$ sessions is proportional to $(M+\alpha)$ rather than $M(1+\alpha)$ as in the non-adaptive CS-based feedback scheme elaborated in Section III. Hence, as compared to the non-adaptive CS-based feedback scheme, the feedback load for the hybrid CS-KLT method proposed here is reduced by nearly $M$-fold in a quasistationary system, where $\alpha$ is anticipated to be large.

We can find out how likely that the proposed scheme would reset under different mobility levels (characterized by the Doppler frequency, $f_{D}$ ). In particular, to model the timevarying channel, we presume that $\boldsymbol{H}_{i i d}$ in (1) is evolved over time according to:

$$
\boldsymbol{H}_{i i d, n e w}=\rho \boldsymbol{H}_{i i d}+\sqrt{1-\rho^{2}} \boldsymbol{Z},
$$

where $Z$ is an $N_{r} \times N_{t}$ matrix of i.i.d. zero-mean com- 


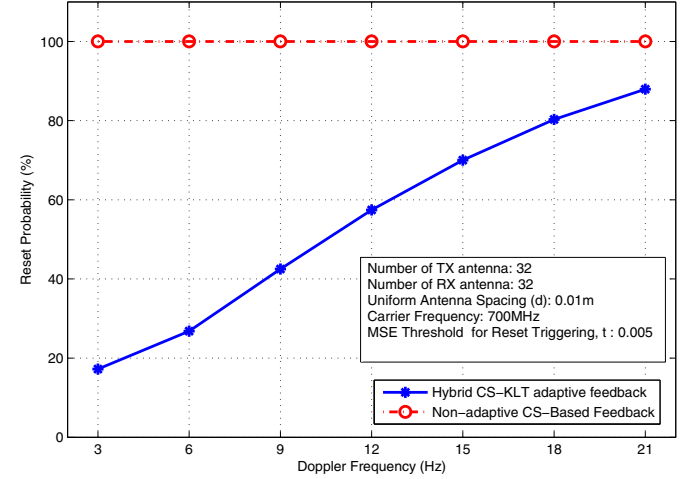

Fig. 6. The reset probabilities of the hybrid CS-KLT adaptive feedback scheme under different mobility levels.

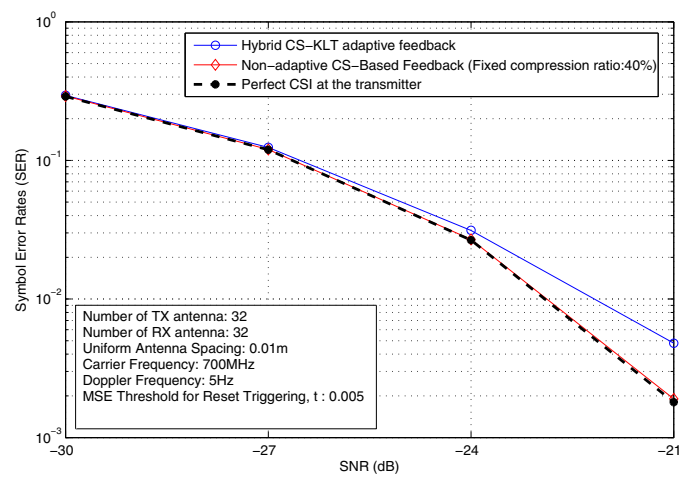

Fig. 7. Rank-1 beamforming performance comparisons between the nonadaptive CS-based feedback scheme with a fixed compression ratio (40\%) and the hybrid CS-KLT adaptive feedback scheme.

plex Gaussian random entries with unit variance, and $\rho=$ $J_{0}\left(2 \pi f_{D} \tau\right)$ is the time-correlation coefficient with $\tau=1 \mathrm{~ms}$ representing the time displacement between two adjacent signaling sessions. Referring to Figure 6 as the example, the simulation results show that the reset probability with a MSE threshold of $t=0.005$ is below $50 \%$ for $f_{D}<10 \mathrm{~Hz}$. As $f_{D}$ increases, the advantages of the proposed protocol gradually diminish. Although CSI recovery based on the inverse-KLT using (13) is an approximation due to inevitable channel fluctuations, by comparing the performance curve with nonadaptive CS-based feedback in Figure 7 for the case of $f_{D}=$ $5 \mathrm{~Hz}$, we can see that the resultant performance loss caused by the approximation error is small. Remarkably, the performance curve with perfect CSI at the transmitter is also aligned, suggesting that the CS-based feedback compression method enables almost exact channel recovery. The proposed hybrid method can be easily adapted to frequency domain channel feedback, as the channel responses over successive OFDM subcarriers, for example, are usually highly correlated. That is, the feedback with $M$ CS measurements is applied in one of the subcarriers, while $s$ is sent on feedback in several of its neighboring subcarriers.

\section{CONCLUSIONS}

This paper suggests using CS to reduce the burden of CSI feedback in spatially-correlated Massive-MIMO systems. The feedback encoding can be carried out simply by random projections, and highly-accurate channel information recovery can be achieved with a low compression ratio (Figure 2). By dynamically adjust the compression ratio based on the instantaneous link quality, we can strike a balance between performance and feedback overhead (Section IV-A). For slowvarying channels, a hybrid feedback compression method of CS and KLT has been devised (Section IV-B), which leverages the optimality property of the KLT basis to further improve CSI feedback efficiency. We conclude that CS is a flexible and promising approach to design the CSI feedback mechanisms for Massive-MIMO systems. In bid to implement the proposed mechanisms in real systems, many practical issues that we did not consider, such as channel estimation errors, quantization noise, and radio impairments in the feedback link, should be addressed in future studies.

\section{REFERENCES}

[1] P. Judge. (2011) GreenTouch shows low power wireless. eWeek Europe. [Online]. Available: http://www.eweekeurope.co.uk/news/greentouchshows-low-power-wireless-19719

[2] T. L. Marzetta, "Noncooperative cellular wireless with unlimited numbers of base station antennas," IEEE Trans. Wireless Commun., vol. 9, pp. 3590-3600, Nov. 2010.

[3] J. Jose, A. Ashikhmin, P. Whiting, and S. Vishwanath, "Channel estimation and linear precoding in multiuser multiple-antenna TDD systems," IEEE Trans. Veh. Technol., vol. 60, pp. 2102-2116, June 2011.

[4] J. Hoydis, S. ten Brink, and M. Debbah, "Massive MIMO: how many antennas do we need?" July 2011, http://arxiv.org/abs/1107.1709.

[5] D. J. Love, R. W. Heath, V. K. N. Lau, D. Gesbert, B. D. Rao, and M. Andrews, "An overview of limited feedback in wireless communication systems," IEEE J. Select. Areas Commun., vol. 26, pp. 1341-1365, Oct. 2008.

[6] E. Candes, J. Romberg, and T. Tao, "Robust uncertainty principles: Exact signal reconstruction from highly incomplete frequency information," IEEE Trans. Inform. Theory, vol. 52, pp. 489-509, Feb. 2006.

[7] R. G. Baraniuk, "Compressive sensing," IEEE Signal Processing Mag., vol. 24, pp. 118-124, July 2007.

[8] Y. Li and R. Song, "Novel schemes of CQI feedback compression based on compressive sensing for adaptive OFDM transmission," KSII Trans. Internet and Info. Systems, vol. 5, pp. 703-719, Apr. 2011.

[9] S. T. Qaseem, T. Y. Al-Naffouri, M. E. Eltayeb, and H. R. Bahrami, "Compressive sensing for feedback reduction in MIMO broadcast channels," July 2009, submitted to IEEE. Trans. Wireless Commun.

[10] L. M. Davis, S. V. Hanly, P. Tune, and S. R. Bhaskaran, "Multi-antenna downlink broadcast using compressed-sensed medium access," in Proc. IEEE Int. Conf. Commun. (ICC), Cape Town, South Africa, May 2010.

[11] T. Yoo, N. Jindal, and A. Goldsmith, "Multi-antenna downlink channels with limited feedback and user selection," IEEE J. Select. Areas Commun., vol. 25, pp. 1478-1491, 2007.

[12] V. R. Cadambe and S. A. Jafar, "Interference alignment and degrees of freedom of the $K$-user interference channel," IEEE Trans. Inform. Theory, vol. 54, pp. 3425-3441, Aug. 2008.

[13] J. A. Tropp and A. C. Gilbert, "Signal recovery from random measurements via orthogonal matching pursuit," IEEE Trans. Inform. Theory, vol. 53, pp. 4655-4666, Dec. 2007.

[14] Y. Rivenson and A. Stern, "Compressed imaging with a separable sensing operator," IEEE Signal Processing Lett., vol. 16, pp. 449-452, June 2009.

[15] J. E. Fowler, "Compressive-projection principal component analysis," IEEE Trans. Image Processing, vol. 18, pp. 2230-2242, Oct. 2009.

[16] Y. Gwon, H. T. Kung, and D. Vlah, "Compressive sensing with directly recoverable optimal basis and applications in spectrum sensing," submitted to IEEE INFOCOM 2012. 